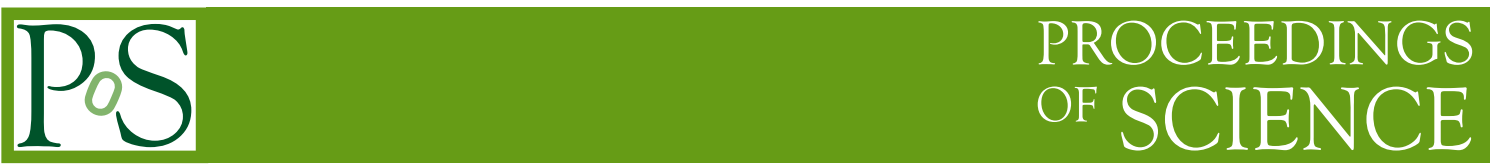

\title{
Constraints on the origin of the UHECR dipole anisotropy outside the Galaxy
}

\author{
Alena Bakalová, ${ }^{a}{ }^{*}$ Petr Trávníček ${ }^{a}$ and Jakub Vícha ${ }^{a}$ \\ ${ }^{a}$ Insitute of Physics of the Czech Academy of Sciences, \\ Na Slovance 1999/2, Prague, Czech Republic \\ E-mail: bakalova@fzu.cz
}

The dipole anisotropy of ultra-high energy cosmic rays above $8 \mathrm{EeV}$ detected by the Pierre Auger Observatory indicates an extragalactic origin of these particles. However, these particles travel long time until they reach the Earth and they are deflected by magnetic fields they cross during their propagation. Therefore, the amplitude and direction of the observed dipole on Earth necessarily does not need to correspond to the anisotropy of the flux when it enters our Galaxy. We present an analysis of influence of the Galactic magnetic field on arrival directions of cosmic rays using numerical simulations within the CRpropa3 package. Jansson-Farrar model of the Galactic magnetic field is used to propagate particles inside the Galaxy. We investigate possible directions and amplitudes of the dipole outside the Galaxy for different mass composition scenarios so that the final direction and amplitude on Earth is compatible with the measured dipole.

40th International Conference on High Energy physics - ICHEP2020

July 28 - August 6, 2020

Prague, Czech Republic (virtual meeting)

\footnotetext{
${ }^{*}$ Speaker
} 


\section{Motivation and method}

Recent measurements of the Pierre Auger Observatory (Auger) indicate that cosmic rays with energies above $8 \mathrm{EeV}$ exhibit a dipole anisotropy in arrival directions [1]. Direction of this dipole with amplitude $A=6.5_{-0.9}^{+1.3} \%$ is far $\left(\sim 125^{\circ}\right)$ from the Galactic center suggesting that these particles are of an extragalactic origin. In this work, we investigate possible features of such dipole anisotropy at the border of the Galaxy before cosmic rays enter the Galactic magnetic field (GMF).

In order to find possible extragalactic direction of the dipole and its initial amplitude $A_{0}$ a series of direct simulations of cosmic rays propagating in the Jansson-Farrar model of the GMF (JF12) [2] was performed in the CRpropa 3 [3]. An isotropic flux was simulated for four primary particles; $\mathrm{H}$, $\mathrm{He}, \mathrm{N}$ and $\mathrm{Fe}$ with the power law energy spectrum of spectral index $\gamma=3$ for energies (8-100) EeV. Particles are collected on an observer with radius $100 \mathrm{pc}$ placed at $8.5 \mathrm{kpc}$ from the Galactic center.

Cosmic rays hitting the observer were reweighted according to their positions at the galaxy border to follow a given dipole distribution. The whole space of different extragalactic directions of the dipole in longitude and latitude with a step of $1^{\circ}$ was investigated for single primary and mixed composition scenarios with a step of $10 \%$ in composition fractions for initial amplitudes from $6.5 \%$ up to $20 \%$. Parameters of the injected amplitude and extragalactic directions of the dipole for given composition mix are selected as possible solutions, if the reconstructed amplitude and direction of the dipole on the observer are compatible with the Auger measurement within $1 \sigma$.

\section{Results}

Concerning the single primary scenarios, results for pure $\mathrm{H}$ and pure $\mathrm{He}$ are suggesting similar dipole attributes. Possible extragalactic directions of the dipole are found for initial amplitudes $A_{0}<10 \%$ for both primaries while larger initial amplitudes generate excessive amplitudes on the observer compared to the measurement. The areas of extragalactic directions of the dipole leading to compatible amplitudes and directions of the dipole on the observer are depicted on the left panel in Figure 1 for $\mathrm{H}$ with $A_{0}=8 \%$. The intersection of these two areas represents a set of possible extragalactic directions of the dipole for given $A_{0}$.

The area of possible extragalactic directions of the dipole is much smaller in the case of pure $\mathrm{N}$ or Fe composition. There are few solutions found for pure $\mathrm{N}$ composition with $A_{0}>14 \%$. However, the effect of isotropisation of cosmic rays in the GMF is so strong for pure Fe composition that even for $A_{0}=20 \%$ the amplitude of the dipole on the observer is extremely low compared to the measurement and consequently no solutions are found.

The number of possible extragalactic directions of the dipole strongly depends on the initial amplitude in the case of a mixed composition. Different mass composition mixes require specific initial amplitudes to describe the data well. Generally, a heavier composition mix requires a higher $A_{0}$ as the isotropisation of cosmic rays in the GMF is stronger for lower rigidities, while for a light composition rather low $A_{0}$ is needed. An area of possible extragalactic directions of the dipole is shown on the right panel in Figure 1 for mixed composition of $20 \% \mathrm{He}$ and $80 \% \mathrm{~N}$ and initial amplitude $A_{0}=10 \%$. The number of possible extragalactic directions of the dipole with respect to the fraction of light nuclei is depicted in Figure 2 for different $A_{0}$ (left: proton fraction $f_{H}$ for $\mathrm{H}+\mathrm{N}$ composition mix, right: fraction of $\mathrm{H}+\mathrm{He} f_{H+H e}$ for composition mix of all four elements). As well as in the case of single primary scenario, the possible extragalactic directions of the dipole are located within $\sim 30^{\circ}$ from the direction of the dipole observed by Auger. 

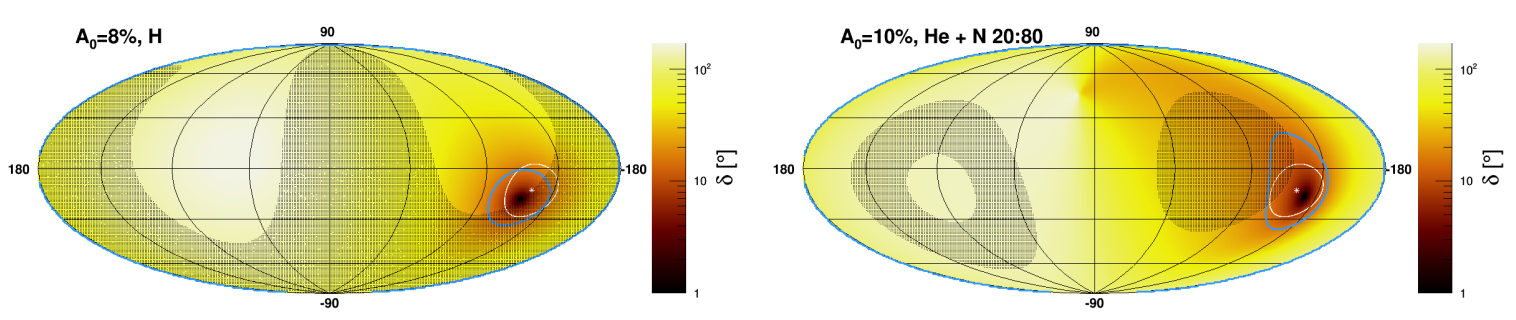

Figure 1: Directions of the dipole outside the Galaxy compatible at $1 \sigma$ level with measurement of the dipole direction (blue contour) and amplitude (dotted area) for $\mathrm{H}$ with $A_{0}=8 \%$ (left) and $\mathrm{He}+\mathrm{N}$ with $A_{0}=10 \%$ (right). $\delta$ shows the angular distance of the reconstructed dipole on the observer and the measured one.
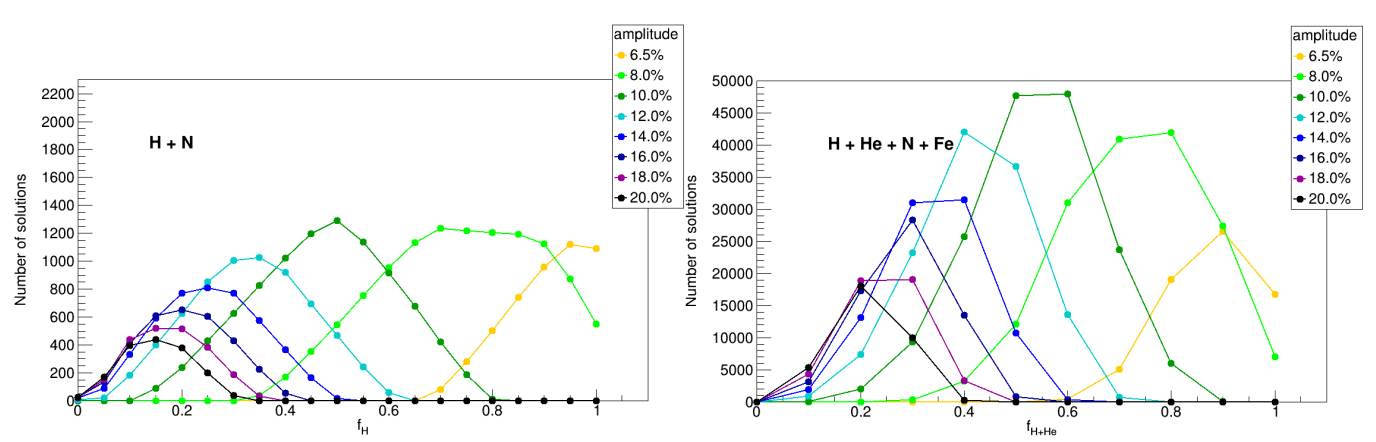

Figure 2: Number of possible extragalactic directions $\left(1^{\circ} \times 1^{\circ}\right.$ grid $)$ depending on the fraction of light elements for different initial amplitudes $A_{0}$. Composition mix: $\mathrm{H}+\mathrm{N}$ (left), $\mathrm{H}+\mathrm{He}+\mathrm{N}+\mathrm{Fe}$ (right).

\section{Conclusions}

We show the influence of the GMF on the dipole distribution of cosmic rays above $8 \mathrm{EeV}$. Different initial directions and amplitudes of the dipole outside the Galaxy were investigated for various mass compositions. Most of the possible extragalactic directions of the dipole compatible with the measurement are located in a region within $30^{\circ}$ from the measured direction by Auger. The observed anisotropy can originate from both single primary and mixed composition scenarios, however, single iron primaries cannot create a dipole compatible with the measurement.

Acknowledgments: This work was supported by: Czech Republic - Grant No. MEYS LTT18004, LM2018102, CZ.02.1.01/0.0/0.0/16 13/0001402.

\section{References}

[1] The Pierre Auger Collaboration, Observation of a large-scale anisotropy in the arrival directions of cosmic rays above $8 \cdot 10^{18} \mathrm{eV}$, Science 357.6357 (2017) [ astro-ph/1709.07321v1].

[2] R. Jansson and G. R. Farrar, A New Model of the Galactic Magnetic Field, ApJ 757.1 (2012) 14 [astro-ph/1204.3662v1].

[3] R. Alves Batista et al., CRPropa 3-a public astrophysical simulation framework for propagating extraterrestrial ultra-high energy particles, JCAP 05 (2016) 038 [astro-ph/1603.07142v2]. 